\title{
Study of fetal and postnatal morphological development of the brain sulci
}

\author{
Laboratory investigation
}

\author{
Koshiro Nishikuni, M.D., and Guilherme Carvalhal Ribas, M.D. \\ Clinical Anatomy Discipline, Department of Surgery-Medical Investigation Laboratory 02, University of São \\ Paulo Medical School; and Hospital Albert Einstein, São Paulo, Brazil
}

\begin{abstract}
Object. The surface of the developing fetal brain undergoes significant morphological changes during fetal growth. The purpose of this study was to evaluate the morphological development of the brain sulci from the fetal to the early postnatal period.

Methods. Two hundred fourteen brain hemispheres from 107 human brain specimens were examined to evaluate the timing of sulcal formation, from its appearance to its complete development. These brains were obtained from cadavers ranging in age from 12 weeks of gestation to 8 months of postnatal life.

Results. The order of appearance of the cerebral sulci, and the number and percentages of specimens found in this study were as follows: longitudinal cerebral fissure at 12 weeks $(10 / 10,100 \%)$; callosal sulcus at 12 weeks (10/10, 100\%); hippocampal sulcus at 15 weeks $(7 / 10,70 \%)$; lateral sulcus at 17 weeks $(20 / 22,90.9 \%)$; circular insular sulcus at 17 weeks $(18 / 22,81.8 \%)$; olfactory sulcus at 17 weeks $(18 / 22,81.8 \%)$; calcarine sulcus at 17 weeks $(14 / 22,63.6 \%)$; parietooccipital sulcus at 17 weeks $(11 / 22,50 \%)$; cingulate sulcus at 19 weeks $(16 / 20,80 \%)$; central sulcus at 21 weeks $(22 / 38,57.9 \%)$; orbital sulcus at 22 weeks $(9 / 16,56.2 \%)$; lunate sulcus at $24 \pm 2$ weeks $(12 / 16$, $75 \%)$; collateral sulcus at $24 \pm 2$ weeks $(8 / 16,50 \%)$; superior frontal sulcus at $25 \pm 2$ weeks $(5 / 6,83.3 \%)$; rhinal sulcus at $25 \pm 2$ weeks $(3 / 6,50 \%)$; precentral sulcus at $26 \pm 3$ weeks $(2 / 4,50 \%)$; postcentral sulcus at $26 \pm 3$ weeks $(2 / 4,50 \%)$; superior temporal sulcus at $26 \pm 3$ weeks $(2 / 4,50 \%)$; central insular sulcus at $29 \pm 2$ weeks $(4 / 4,100 \%)$; intraparietal sulcus at $29 \pm 2$ weeks $(2 / 4,50 \%)$; paraolfactory sulcus at $29 \pm 2$ weeks $(2 / 4,50 \%)$; inferior frontal sulcus at $30 \pm 3$ weeks $(2 / 4,50 \%)$; transverse occipital sulcus at $30 \pm 3$ weeks $(2 / 4,50 \%)$; occipitotemporal sulcus at $30 \pm 3$ weeks $(2 / 4,50 \%)$; marginal branch of the cingulate sulcus at $30 \pm 3$ weeks $(2 / 4,50 \%)$; paracentral sulcus at $30 \pm 3$ weeks $(2 / 4,50 \%)$; subparietal sulcus at $30 \pm 3$ weeks $(2 / 4,50 \%)$; inferior temporal sulcus at $31 \pm 3$ weeks $(3 / 6,50 \%)$; transverse temporal sulcus at $33 \pm 3$ weeks $(6 / 8,75 \%)$; and secondary sulcus at $38 \pm 3$ weeks $(2 / 4,50 \%)$.

Conclusions. The brain is subjected to considerable morphological changes throughout gestation. During fetal brain development the cortex begins to fold in, thereby increasing the cortical surface. All primary sulci are formed during fetal life. The appearance of each sulcus follows a characteristic timing pattern, which may be used as one of the reliable guides pertinent to gestational age and normal fetal development.
\end{abstract}

(http://thejns.org/doi/abs/10.3171/2012.9.PEDS12122)

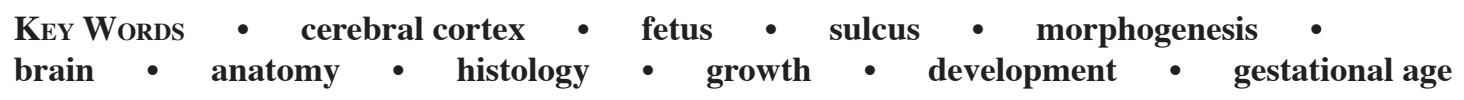

$\mathrm{M}$ ODERN microneurosurgery has made the sulci and fissures the main landmarks of the brain surface, and also has made them possible microneurosurgical corridors into its depth. ${ }^{37-39}$ Although they have a very well-defined general organization, ${ }^{22,24,27,32,33}$ the sulci are usually difficult to identify on neuroimaging studies, in fixed anatomical specimens, and during neurosurgical exposures, even with the aid of neuronavigation systems. The sulci are difficult to identify, not only due to their arachnoidal and occasional vascular coverings, but more particularly due to their common interruptions and anatomical variations, ${ }^{24,27}$ which continue to lead neuro- radiologists and neurosurgeons to search for a better understanding of their anatomical features.

Because the sulci of the human brain are specifically the result of an evolutionary infolding process ${ }^{24,29}$ that is repeated throughout embryological and fetal development ${ }^{5,21,36}$ (Fig. 1) and because this topic has scarcely been examined, ${ }^{5}$ the study of their embryological appearance can be of great assistance, not only for a better general

This article contains some figures that are displayed in color online but in black-and-white in the print edition. 


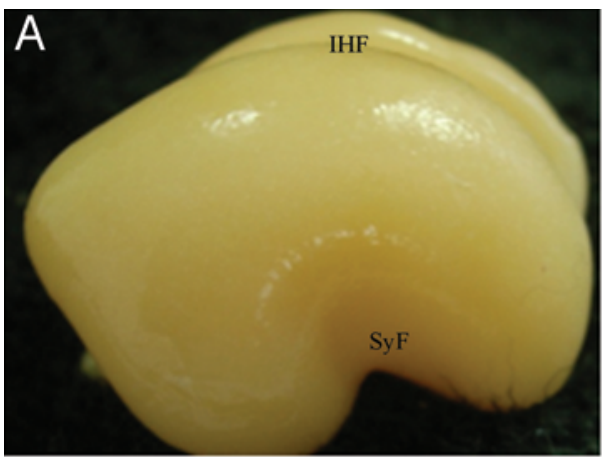

17 weeks

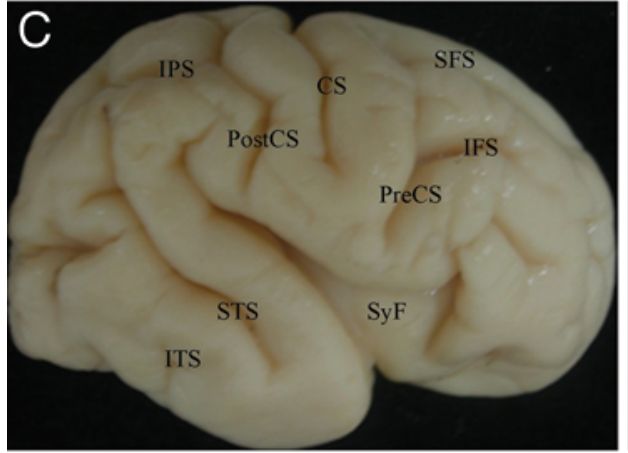

36 weeks

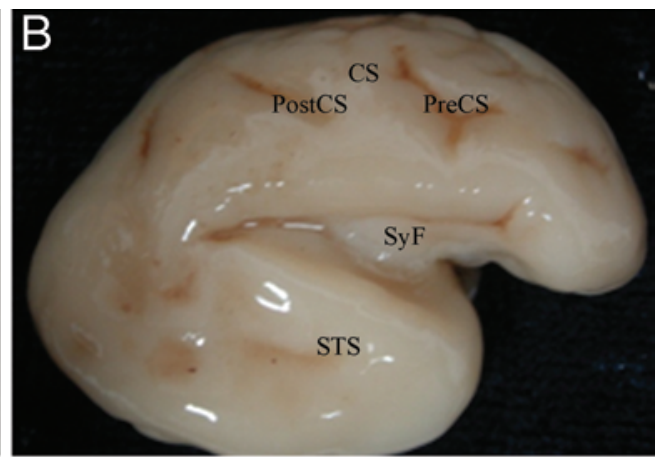

24 weeks

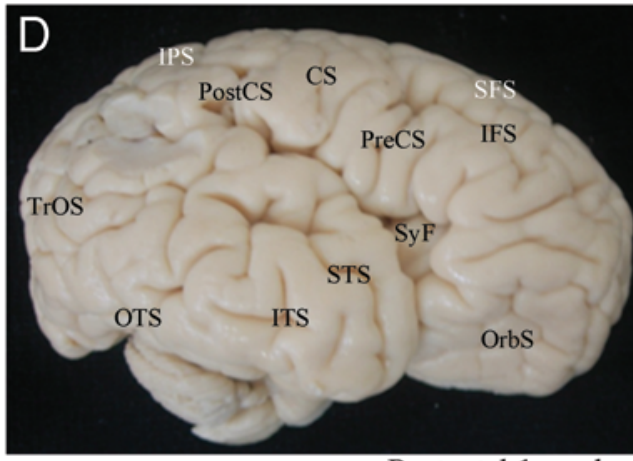

Postnatal 1 week

FiG. 1. Development of sulci of the superolateral cerebral surface in the fetus at 17 (A), 24 (B), and 36 weeks (C), and at 1 postnatal week (D). CS = central sulcus; IFS = inferior frontal sulcus; IHF = interhemispheric fissure (longitudinal cerebral fissure); IPS = intraparietal sulcus; ITS = inferior temporal sulcus; OrbS = orbital sulcus; OTS = occipitotemporal sulcus; PostCS = postcentral sulcus; PreCS = precentral sulcus; SFS = superior frontal sulcus; STS = superior temporal sulcus; SyF = lateral sulcus (sylvian fissure); $\operatorname{TrOS}=$ transverse occipital sulcus.

understanding of the anatomy, but also for dealing with practical gestational, neuroradiological, and microneurosurgical issues.

\section{Methods}

Two hundred fourteen cerebral hemispheres obtained from 107 fetal cadavers were examined at the Division of Pathological Anatomy and at the Death Verification Service of the University of São Paulo Medical School, as previously authorized by the Ethical Committee for Analysis of Research Projects. Removal of brains was performed using a typical autopsy technique. Specimens with congenital malformations and or structural damage were excluded.

The removed brains were fixed in $10 \%$ formalin solution for a period of at least 1 month, and then the arachnoidal membranes and superficial vessels of the cerebral hemispheres were removed to simplify the identification of the sulci on the superolateral, medial, and inferior brain surfaces. Photographs of anatomical dissections were obtained using a Nikon Coolpix 5200 5.1-megapixel digital camera.

The sulci formations were classified as follows: absent, when the cerebral surface was totally smooth; developing, in which points and grooves were considered sulci in the formation phase; and formed, when the clear and complete depressions were considered sulci.
To evaluate the results, examined brains were divided into groups according to the body weights of the fetuses: 1) groups of brains from fetuses, with body weights up to $1000 \mathrm{~g}$ with a weight gap among the groups of $100 \mathrm{~g}$; and 2) groups of brains from fetuses with body weights greater than $1001 \mathrm{~g}$, with a weight gap among the groups of $250 \mathrm{~g}$ (Table 1). Because fetal body weight correlates with gestational age, ${ }^{31}$ when at least $50 \%$ of the cases of a group showed a particular sulcus, this sulcus was classified as formed, and we then considered that particular gestational age to be related to the formation of these specific sulci.

\section{Results}

During the removal of all of the brains, we observed that the longitudinal cerebral fissure was always already formed. The findings according to the fetal body weight groups were as follows:

\section{0-100 grams}

We observed that the callosal sulcus was already formed in $100 \%$ and the hippocampal sulcus in $80 \%$ of the examined specimens.

\section{1-200 grams}

We found complete formation of the hippocampal 
Study of the fetal morphological development of the brain sulci

TABLE 1: Fetal body weight groups used in the study

\begin{tabular}{|c|c|}
\hline Fetal Body Weight Groups (g) & No. of Specimens \\
\hline $1-100$ & 10 \\
\hline $101-200$ & 22 \\
\hline $201-300$ & 20 \\
\hline $301-400$ & 38 \\
\hline $401-500$ & 16 \\
\hline $501-600$ & 6 \\
\hline $601-700$ & 4 \\
\hline $701-800$ & 6 \\
\hline $801-900$ & 4 \\
\hline $901-1000$ & 4 \\
\hline $1001-1250$ & 6 \\
\hline $1251-1500$ & 8 \\
\hline $1501-1750$ & 6 \\
\hline $1751-2000$ & 6 \\
\hline $2001-2250$ & 6 \\
\hline $2251-2500$ & 4 \\
\hline $2501-2750$ & 4 \\
\hline $2751-3000$ & 4 \\
\hline $3001-3250$ & 2 \\
\hline $3251-3500$ & 12 \\
\hline $3501-3750$ & 2 \\
\hline $3751-4000$ & 2 \\
\hline postnatal & 16 \\
\hline total & 208 \\
\hline
\end{tabular}

sulcus and the presence of the lateral and olfactory sulci in more than $80 \%$ of the samples, and presence of the circular insular, calcarine, and parietooccipital sulci in more than half of the specimens.

\section{1-300 grams}

The lateral and circular insular sulci were formed at this body weight in $100 \%$ of the specimens, and the cingulate sulcus was formed in $80 \%$ of the specimens.

\section{1-400 grams}

The formation of the central sulcus was detected in $60 \%$ of the cases in this group.

\section{1-500 grams}

The lunate sulcus was evident in $75 \%$, and the orbital and collateral sulci in more than $50 \%$ of the specimens.

\section{1-600 grams}

The central and calcarine sulci were formed in $100 \%$ of the specimens at this weight, and we observed the formation of the superior frontal sulcus in $80 \%$ and of the rhinal sulcus in $50 \%$ of the specimens.

601-700 grams

The olfactory and parietooccipital sulci were already formed in $100 \%$ of the specimens, and half of the precen- tral, postcentral, and superior temporal sulci were already visible.

\section{1-800 grams}

The transverse occipital sulcus at this weight was evident in $70 \%$ of the specimens.

801-900 grams

The precentral and cingulate sulci were formed in $100 \%$ of the specimens, and several new sulci appeared: on the superolateral surface, the central insular sulcus appeared in $100 \%$ and the intraparietal sulcus in $50 \%$ of the specimens; and on the medial surface, the paraolfactory sulcus was formed in $50 \%$ of specimens.

\section{1-1000 grams}

The superior temporal sulcus was detected in $100 \%$ of the specimens, and the formation of the following sulci were observed in $50 \%$ of the specimens: on the superolateral surface, the inferior frontal and transverse occipital sulci; on the inferior surface, the occipitotemporal sulcus; and on the medial surface, the marginal branch of the cingulate sulcus and the paracentral and subparietal sulci.

1001-1250 grams

The formation of the inferior temporal sulcus was observed in $50 \%$ of the specimens.

\section{1-1500 grams}

Together with the formation of the transverse temporal sulcus observed in $75 \%$, all of the main sulci were already formed in at least $50 \%$ of the examined specimens in this group.

\section{1-1750 grams}

Complete formation of the superior frontal, inferior frontal, postcentral, intraparietal, inferior temporal, transverse temporal, orbital, rhinal, collateral, and marginal branch of the cingulate sulci was found in all specimens.

2001-2250 grams

Together with the formation of the central insular, occipitotemporal, paracentral, paraolfactory and subparietal sulci, all the main sulci were formed in $100 \%$ of the specimens.

\section{1-2500 grams}

The secondary sulci were clearly developed in $50 \%$ of the examined specimens at this weight.

\section{More Than 3250 grams}

In fetuses that weighed more than $3250 \mathrm{~g}$, the main and secondary sulci were fully formed.

During the early postnatal period, no formation of new main sulci was observed. Table 2 summarizes the general findings, listing the development of the fetuses' cerebral fissures and sulci together with their related body weights and respective estimated gestational ages. 
TABLE 2: Development of the cerebral sulci, related body weights, and estimated gestational ages in the specimens

\begin{tabular}{|c|c|c|}
\hline Sulcus & $\begin{array}{c}\text { Fetal Body } \\
\text { Weight Group (g) }\end{array}$ & $\begin{array}{c}\text { Gestational } \\
\text { Age (wks) }\end{array}$ \\
\hline longitudinal cerebral fissure & $20-100$ & 12 \\
\hline \multicolumn{3}{|l|}{ superolateral cerebral surface } \\
\hline lateral sulcus & $101-200$ & 17 \\
\hline circular insular sulcus & $101-200$ & 17 \\
\hline central insular sulcus & $801-900$ & $29 \pm 2$ \\
\hline central sulcus & $301-400$ & 21 \\
\hline precentral sulcus & $601-700$ & $26 \pm 3$ \\
\hline superior frontal sulcus & $501-600$ & $25 \pm 2$ \\
\hline inferior frontal sulcus & $901-1000$ & $30 \pm 3$ \\
\hline postcentral sulcus & $601-700$ & $26 \pm 3$ \\
\hline intraparietal sulcus & $801-900$ & $29 \pm 2$ \\
\hline transverse occipital sulcus & $901-1000$ & $30 \pm 3$ \\
\hline lunate sulcus & $401-500$ & $24 \pm 2$ \\
\hline superior temporal sulcus & $601-700$ & $26 \pm 3$ \\
\hline inferior temporal sulcus & $1001-1250$ & $31 \pm 3$ \\
\hline transverse temporal sulcus & $1251-1500$ & $33 \pm 3$ \\
\hline \multicolumn{3}{|l|}{ inferior cerebral surface } \\
\hline olfactory sulcus & $101-200$ & 17 \\
\hline orbital sulcus & $401-500$ & 22 \\
\hline hippocampal sulcus & $20-100$ & 15 \\
\hline rhinal sulcus & $501-600$ & $25 \pm 2$ \\
\hline collateral sulcus & $401-500$ & $24 \pm 2$ \\
\hline occipitotemporal sulcus & $901-1000$ & $30 \pm 3$ \\
\hline \multicolumn{3}{|l|}{ medial cerebral surface } \\
\hline callosal sulcus & $20-100$ & 12 \\
\hline cingulate sulcus & $201-300$ & 19 \\
\hline marginal branch of cingulate sulcus & $901-1000$ & $30 \pm 3$ \\
\hline paracentral sulcus & $901-1000$ & $30 \pm 3$ \\
\hline paraolfactory sulcus & $801-900$ & $29 \pm 2$ \\
\hline subparietal sulcus & $901-1000$ & $30 \pm 3$ \\
\hline calcarine sulcus & $101-200$ & 17 \\
\hline parietoccipital sulcus & $101-200$ & 17 \\
\hline secondary sulci & $2251-2500$ & $38 \pm 3$ \\
\hline
\end{tabular}

\section{Discussion}

Although humankind has long been interested in the human brain, ${ }^{24,26}$ it is interesting to point out that knowledge that the brain sulci and gyri have a general arrangement dates to less than 160 years ago..$^{22,32,33}$ In addition to describing the optic radiation, French anatomist Louis Pierre Gratiolet (1815-1865) $)^{32}$ distinguished the primary and secondary sulci based on their respective evolutionary appearances, created the concept of cerebral lobes by adopting the terms previously proposed by Frederich Arnold in relation to the skull bones, coined the term "plis de passage" to describe the connections between adjacent gyri, and was the first anatomist to perceive that the brain sulci and gyri were not chaotically arranged. ${ }^{22,32,33}$

Essentially the human brain is organized as follows: superolaterally, the frontal and temporal regions of each hemisphere are each composed of 3 horizontal gyri; the central region is composed of 2 slightly oblique gyri; the parietal region is composed of 2 lobules, with a quadrangular superior lobule and an inferior lobule consisting of 2 semicircular gyri; the occipital region is composed of vertical and horizontal gyri, in which the superior occipital gyrus is always present and vertical, the middle occipital gyrus is not always present, and the inferior occipital gyrus is always present and horizontal; the insula is composed of 4-5 diagonal gyri. While the superior frontal, inferior frontal, superior temporal, and inferior temporal sulci separate the 3 frontal and temporal gyri, respectively, the lateral fissure (sylvian fissure) divides the frontal from the temporal lobe, the intraparietal sulcus separates the superior from the inferior parietal lobule, and the superior and inferior (or transverse) occipital sulci separate the occipital gyri. ${ }^{24,33,36}$

Medially, the external lateral gyri extend along the superior and inferolateral borders of each hemisphere. Together, these gyri constitute an outer medial ring, which surrounds a more well-defined C-shaped inner ring primarily composed of 2 continuous gyri, with these 2 rings separated by the cingulate, subparietal, and collateral sulci. ${ }^{24,33,36}$

Inferiorly, the base of each hemisphere consists of 2 horizontal gyri longitudinally oriented between the lateral extended gyri (along the inferolateral and inferomedial borders) and the medial continuous gyri of the inner ring, separated laterally by the occipitotemporal sulcus and medially by the collateral sulcus. ${ }^{24,33,36}$

Human brain sulci developed mainly due to an evolutionary infolding process that caused a progressive increase in the cerebral cortical surface without a proportional increase in brain volume. . $25,29,36$ Phylogenetically, the first hemispheric sulcus to appear was the hippocampal sulcus, which separates the archicortex (dentate gyri of the hippocampus) from its paleocortical surrounding structures, including the parahippocampal subiculum, which arises during the phylogenetic and embryological caudal migration of the original supracallosal hippocampus. ${ }^{29}$ The second hemispheric sulcus was the rhinal sulcus, which demarcates the border between the paleocortex and neocortex, and that appeared due to the ventral displacement of the neocortex. ${ }^{29}$ In humans, the rhinal sulcus then separates the parahippocampal uncus from the rest of the neocortical temporal lobe. The hippocampal sulcus and rhinal sulcus were both already present in early mammals. ${ }^{24,29}$

Embryologically, the sulci develop according to a sequence that reflects their phylogeny and a hierarchy that exists among them. Their formation begins with the appearance of the fissures, followed by sulci related to eloquent areas of the brain, and finally with the secondary and tertiary cortical area sulci., ${ }^{4,5,20,22}$ The fissures correspond to the more well-developed and anatomically constant sulci, and the gyri or convolutions that have a more rounded or quadrangular shape are usually referred

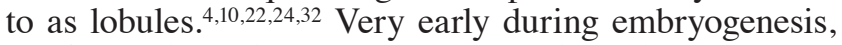
the forebrain vesicle (or prosencephalic vesicle, the most superior of the 3 primary brain vesicles originating from the neural tube) divides into the endbrain (telencephalic) 


\section{Study of the fetal morphological development of the brain sulci}

and interbrain (diencephalic) vesicles. ${ }^{30}$ By approximately the 10th week of gestation, a superior midline depression of the endbrain gives rise to the interhemispheric fissure, and the transverse fissure of the brain appears between the endbrain and interbrain vesicles. ${ }^{7,30}$

At the end of the 3rd month the superolateral surface of the cerebral hemisphere shows a slight depression anterosuperior to the temporal pole. This depression corresponds to the site of the corpus striatum in the floor and lateral wall of the ventricle, and its presence is due to the more rapid growth of the adjoining cortical regions. ${ }^{36}$ This lateral cerebral fossa gradually becomes overlapped and submerged by the frontal and temporal opercula, and is progressively converted into the lateral cerebral sulcus; its floor becomes the insula. The process, however, is not completed in its most anterior part until after birth. ${ }^{36}$

The lateral sulcus, or sylvian fissure, then results from the growth of the infolding opercula of the surrounding lobes, overlapping onto the insula, and it became narrow only in humans, in whom the frontoparietal operculum is particularly developed. ${ }^{29,30}$ The most notable development was that of the pars triangularis and pars opercularis of the inferior frontal gyrus, which, in the dominant hemisphere of the human brain, correspond to the Broca area. ${ }^{10}$

Between the 8th and 10th weeks of gestation, transitory furrows that are not precursors of the permanent sulci appear in the cerebral hemispheric surfaces. These furrows persist until the 5th month, when the brain surfaces become smooth and the insular area is the only evident depression. ${ }^{21,36}$

During the 4th and 5th months of gestation the first identifiable sulci (olfactory, calcarine, parietooccipital, cingulate, and central) begin to appear, followed by additional secondary and tertiary furrows, some of which develop only after birth. ${ }^{5,21}$

In Gray's Anatomy, ${ }^{36}$ Williams and Warwick summarize the development of brain sulci during the embryological and fetal cycle as follows: during the 5th gestational month the sulcus cinguli appears on the medial aspect of the hemisphere, but it is not until the 6th month that sulci appear on the inferior and superolateral aspects. The central, precentral, and postcentral sulci each appear in 2 parts, upper and lower, which usually coalesce shortly afterward, although they may remain discontinuous. The superior and inferior frontal, intraparietal, occipital, temporal, and collateral sulci make their appearance during the same period, and by the end of the 8th gestational month all of the important sulci can already be recognized.

The brain sulci evolve mainly through an infolding process, which takes place while the entire developing brain undergoes a process of circular curvature, effectively wrapping the thalami in its morphological center. Therefore, it is noteworthy that in their final presentation, the sulci of the superolateral and inferior surfaces of the cerebral hemisphere are directed toward each respective nearest portion of the lateral ventricle. The development of the sulcal pattern of the medial surfaces appears to be particularly influenced by the development of the corpus callosum, because its congenital absence is linked to the absence of an arched cingulate gyrus and to a radial pat- tern of the medial surface sulci. ${ }^{21,24,29,30,36}$ The process of sulcal development and its relatively variable final result are in great part definitely determined by genetics. ${ }^{30}$

Regarding the extensive variability of the basic arrangement of the cortical sulci and gyri, Régis et al..$^{23}$ more recently proposed that the occurrence of such variations might depend on the variable development of connecting gyri buried in the depth of the sulci (plis de passage). Taking into account these buried gyri, and using a database of MR images of 20 healthy patients, these authors proposed an interesting generic model of folding patterns based on a constant number of indivisible units they termed "sulcal roots," presupposing then a common constant protomap. According to this hypothesis, the burying process is believed to result from a tradeoff between the various folding pressures that occur during brain growth, with the superficial variability resulting from the chaotic behavior given by the larger or smaller development of the buried gyral connections, with a major development then causing an interruption of its related sulcus. ${ }^{6}$ Based on this hypothesis, the occasional interruption of the central sulcus, for example, is then due to a more significant development of the middle frontoparietal connection (of Broca) that lies in between the precentral and postcentral gyri at the so-called omega region; ${ }^{3}$ this occurs at the level of the distal end of the superior frontal sulcus ${ }^{24}$ that corresponds to the sulcal root of the central sulcus. ${ }^{6}$

Parallel to further understanding their neuroimaging and microneurosurgical applications, ${ }^{37-39}$ the embryological and fetal development of human brain sulci has been only scarcely studied, ${ }^{5,7,13}$ and is currently of significant interest to also calculate gestational ages with the aid of ultrasonography studies. . $^{23,26,34}$

The development of the main sulci in the embryological, fetal, and postnatal periods are discussed below, according to the order of their appearance.

\section{Longitudinal Cerebral Fissure}

The longitudinal cerebral fissure originates dorsally and gradually develops as a longitudinal invagination of the telencephalic vesicle. It is the first fissure to appear and was observed in the smallest fetus examined; it was also described by Chi et al. ${ }^{5}$ and Levene and Lilford. ${ }^{16}$

\section{Transitory Furrows}

Transitory furrows appear during the initial phase of cerebral development, and then disappear completely, leaving the cerebral surface smooth again. We observed the presence of these furrows in 2 cases.

\section{Sulci of the Superolateral Cerebral Surface}

Lateral Sulcus. The lateral sulcus (Sylvian fissure) is the first to appear on the superolateral surface and evolves in a unique way because phylogenetically it is the sole cerebral sulcus that is not only the result of an invagination process, but also occurs because of a juxtaposition phenomenon of the progressive enlargement of the frontal and temporal lobes (Fig. 1). Embryologically the lateral sulcus is at first a superficial depression in the lateral sur- 
face of the hemispheres that gradually becomes deeper, at the same time as the frontal, parietal, and temporal opercula are also rapidly growing (Fig. 2). This development parallels neuronal migration from the germinal ventricular zone surface to the cortical surface. ${ }^{2}$ The progressive operculization that occurs between the 22nd and 34th gestational weeks characterizes the final aspect of the lateral sulcus at approximately the 36th gestational week. ${ }^{9}$

Circular Insular Sulcus. The structure that constitutes the base of the fossa of the lateral sulcus begins to delimit the lateral surface of the insula, with its superior edge corresponding to the beginning of the circular sulcus of the insula and with its inferior edge initially constituting the lateral sulcus itself. The circular sulcus is initially round, but with the development of the opercula between the 22nd and 24th gestational weeks, it begins to lose its original shape (Fig. 2B-D). An ultrasonography study showed that the angle formed by the insula and the operculum is initially obtuse, and from the 24 th week it becomes acute. ${ }^{34}$

Central Sulcus. In this study we noted that the central sulcus starts as a point or as a groove in the parasagittal region (Fig. 2A and B), and then develops as an evident cortical invagination that extends itself obliquely, inferiorly, and anteriorly, until it reaches the lateral sulcus, and superiorly toward the longitudinal cerebral fissure. The arrows in Fig. 2 illustrate the development of the central sulcus.

Precentral Sulcus. The precentral sulcus develops anterior and roughly parallel to the central sulcus. Unlike the central sulcus, which is usually unbroken, we observed the interruption of the precentral sulcus during cerebral development. According to the findings of Ono et al. ${ }^{21}$ this sulcus is interrupted in $100 \%$ of adults. The postcentral sulcus develops during the same period (Fig. $2 \mathrm{E}$ ), and usually ends either as a continuous sulcus or as 2 segments.

Superior Frontal Sulcus. The formation of the superior frontal sulcus is initially more evident in the frontal pole; it develops running posteriorly until the precentral sulcus (Fig. 2E and F).

Intraparietal Sulcus. The intraparietal sulcus is localized roughly parallel to the longitudinal cerebral fissure, and divides the parietal lobe between the superior and inferior parietal lobules. The most anterior aspect of the intraparietal sulcus usually achieves an arcuate shape; it is inferiorly directed and is frequently continuous with the inferior part of the postcentral sulcus (Fig. 2F).

Transverse Occipital Sulcus. The posterior end of the intraparietal sulcus is localized very close to and sometimes even reaches the transverse occipital sulcus (also called the intraoccipital sulcus ${ }^{8,19}$ or superior occipital sulcus), ${ }^{33}$ which separates the superior occipital gyrus from the middle occipital gyrus. The transverse occipital sulcus is parallel or perpendicular to the interhemispheric fissure, and anteriorly it can be continuous with the intraparietal sulcus (Fig. 2F).
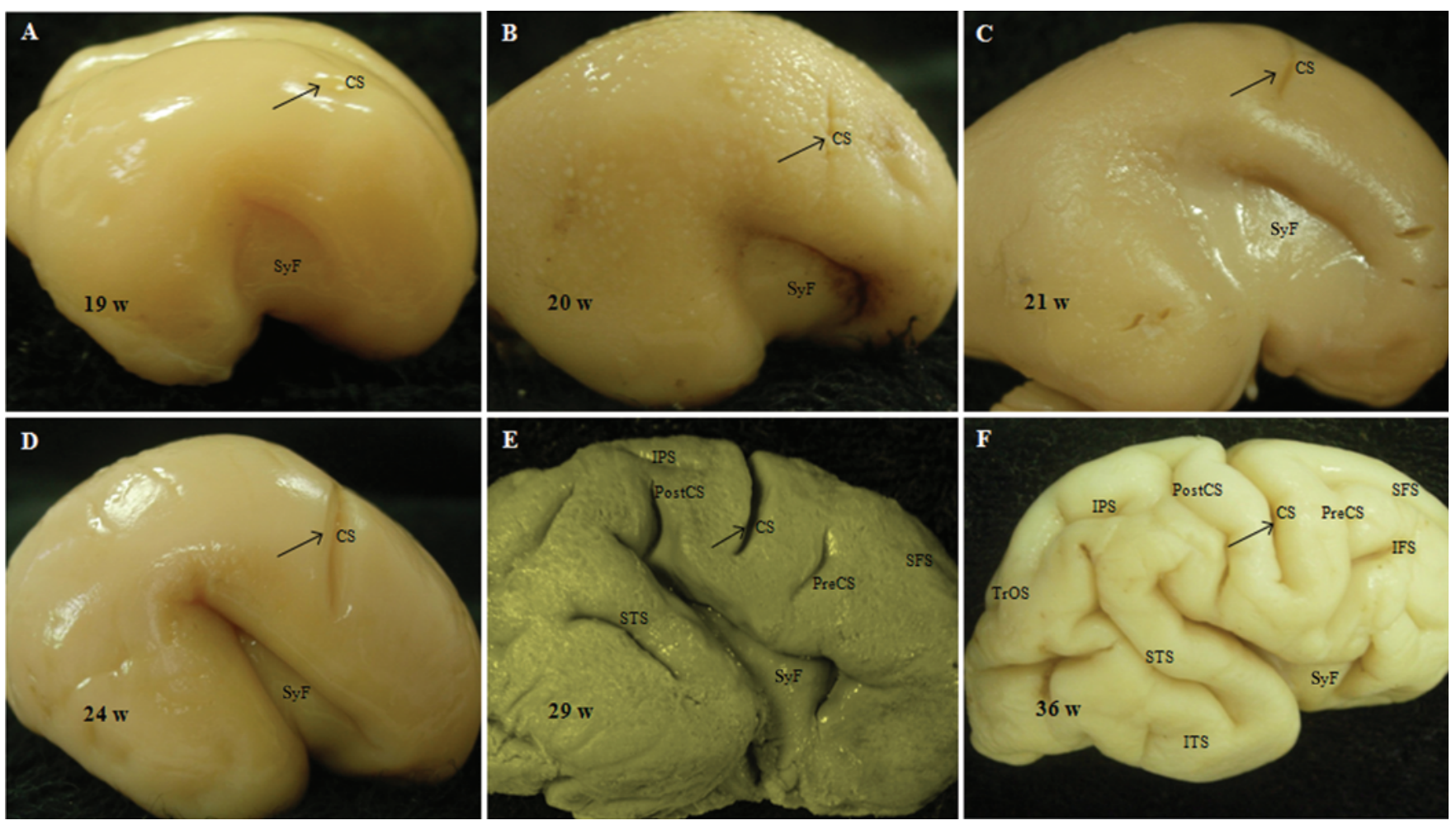

FIG. 2. Development of sulci of the superolateral cerebral surface of the fetus at 19 (A), 20 (B), 21 (C), 24 (D), 29 (E), and 36 weeks (F). The arrows illustrate the central sulci development. $w=$ weeks. 


\section{Study of the fetal morphological development of the brain sulci}

Superior Temporal Sulcus. The superior temporal sulcus, which separates the superior temporal gyrus from the middle temporal gyrus and runs roughly parallel to the lateral sulcus, usually ends as a continuous sulcus with its most distal branch generally terminating inside the angular gyrus, while the inferior temporal sulcus ends as an interrupted sulcus (Fig. 2F) composed of several segments. ${ }^{21}$ Figure 2 illustrates the development of the sulci of the superolateral surface of the brain.

\section{Sulci of the Inferior Cerebral Surface}

Olfactory Sulcus. The olfactory sulcus appears initially as a superficial depression on the medial aspect of the cerebral orbital surface. It then develops parallel to the longitudinal cerebral fissure, underneath the olfactory bulb and tract, and extends, delimiting the rectal gyrus laterally (Fig. 3A-C). According to Dooling et al., ${ }^{6}$ the olfactory sulcus is present in each brain that contains the olfactory bulb and tract and is absent when the olfactory bulb and tract do not develop, which denotes an interesting developmental interrelationship between these structures and that sulcal space.

Hippocampal Sulcus. The hippocampal sulcus corresponds to the first phylogenetic sulcus because it separates the archicortex of the hippocampus from the paleocortex of the subiculum of the parahippocampal gyrus; this separation occurs due to the inferior and lateral displacement of the hippocampus that takes place both evolutionarily and embryologically. ${ }^{29,36}$ Chi et al. ${ }^{5}$ observed that the hippocampal sulcus gradually narrows while the medial portion of the temporal lobe develops. In the present study, the hippocampal sulcus was formed in fetuses heavier than $40 \mathrm{~g}$, at approximately the 15 th week of gestation (Fig. 3A).

Rhinal Sulcus. The rhinal sulcus develops as an evolutionary result of the ventral displacement of the lateral olfactory area or piriform cortex within the development of the anterior temporal neocortex. ${ }^{29}$ Because the lateral olfactory area is constituted from the paleocortex, the rhinal sulcus separates the paleocortex from the neocortex and corresponds to the second sulcus that appears during phylogenesis. In our study, the rhinal sulcus was formed at approximately the 25 th week, and because it is not always easily identified, even in adults, it must be identified as the sulcal space that separates the uncus (paleocortex) from the rest of the temporal pole (neocortex). The collateral sulcus (Fig. 3D-F) may or may not be posteriorly connected to the rhinal sulcus. ${ }^{21}$

Occipitotemporal Sulcus. The occipitotemporal sulcus (Fig. 3D-F) separates the fusiform gyrus from the inferior temporal gyrus ${ }^{21}$ and frequently terminates in the collateral sulcus anteriorly. Figure 3 demonstrates the development of the sulci of the inferior surface of the brain.

\section{Sulci of the Medial Cerebral Surface}

Callosal Sulcus. The callosal sulcus separates the callosal body from the cingulate gyrus. This sulcus was already formed in the lightest fetus we examined (20 g; Fig. 4A), and was therefore considered the first sulcus that appears on the medial surface of the brain. The callosal sulcus develops from the anterior to the posterior direction, concomitantly with the development of the callosal body (Fig. 4A-C). We noted the presence of transitory furrows, which were not related to any forthcoming of the sulci that developed subsequently (Fig. 4A arrows). According to Larroche, ${ }^{14}$ at 12 weeks' gestation all of the cerebral structures are already localized in their expected position except for the callosal body, which develops during the following weeks.

Cingulate Sulcus. The cingulate sulcus develops parallel to the callosal sulcus (Fig. 4C-E), delimiting the cingulate gyrus superiorly. In some fetuses we have found 2 parallel cingulate sulci (Fig. 4F), and in these specimens we have observed that the callosal sulcus was shallower around the genu of the callosal body, as noted by Ono et al. as well. ${ }^{21}$ The final and ascending segment constitutes the marginal branch of the cingulate sulcus, which delineates the paracentral lobule posteriorly. The cingulate sulcus is delineated anteriorly by the paracentral sulcus (Fig. 4E and F), which is formed during the same period as the marginal branch of the cingulate sulcus, and may appear as a side branch of the cingulate sulcus, or emerge from the superolateral surface, or even develop as an anterior extension of the posterior segment of the cingulate sulcus.

Parietooccipital Sulcus. The parietooccipital sulcus divides the parietal lobe from the occipital lobe along the medial cerebral surface, separating the precuneus of the parietal lobe from the cuneus of the occipital lobe (Fig. $4 \mathrm{C}-\mathrm{F}$ ). The development of the parietooccipital sulcus is related to the development of the splenium of the corpus callosum, with the sulcus arising due to the progressive invagination of the medial cerebral surface that contains the occipital and temporal bilateral association fibers. ${ }^{36}$

Calcarine Sulcus. The calcarine sulcus (Fig. 4C-F) generally appears concomitantly with the parietooccipital sulcus, with the emerging point of the latter dividing the calcarine sulcus or fissure into 2 distinct parts. The anterior calcarine segment separates the cingulate isthmus from the lingual gyrus, and the posterior segment, which corresponds to the primary visual cortex, separates the lingual gyrus from the cuneus. Figure 4 illustrates the development of the sulci of the medial surface of the brain.

\section{Secondary Sulci}

The main sulci appear in a relatively short period, generally at the beginning of the third trimester of pregnancy, ${ }^{15}$ and only afterward do the secondary sulci develop. At approximately 34 weeks of gestation (2251-2500 $\mathrm{g}$ of body weight), the secondary sulci begin to develop while the main sulci become deeper. ${ }^{6,35}$

\section{Comparative Analysis of Cerebral Sulci Development}

The formation of the cortical sulci is considered by neuropathologists to be a good marker of cerebral fetal 
K. Nishikuni and G. C. Ribas
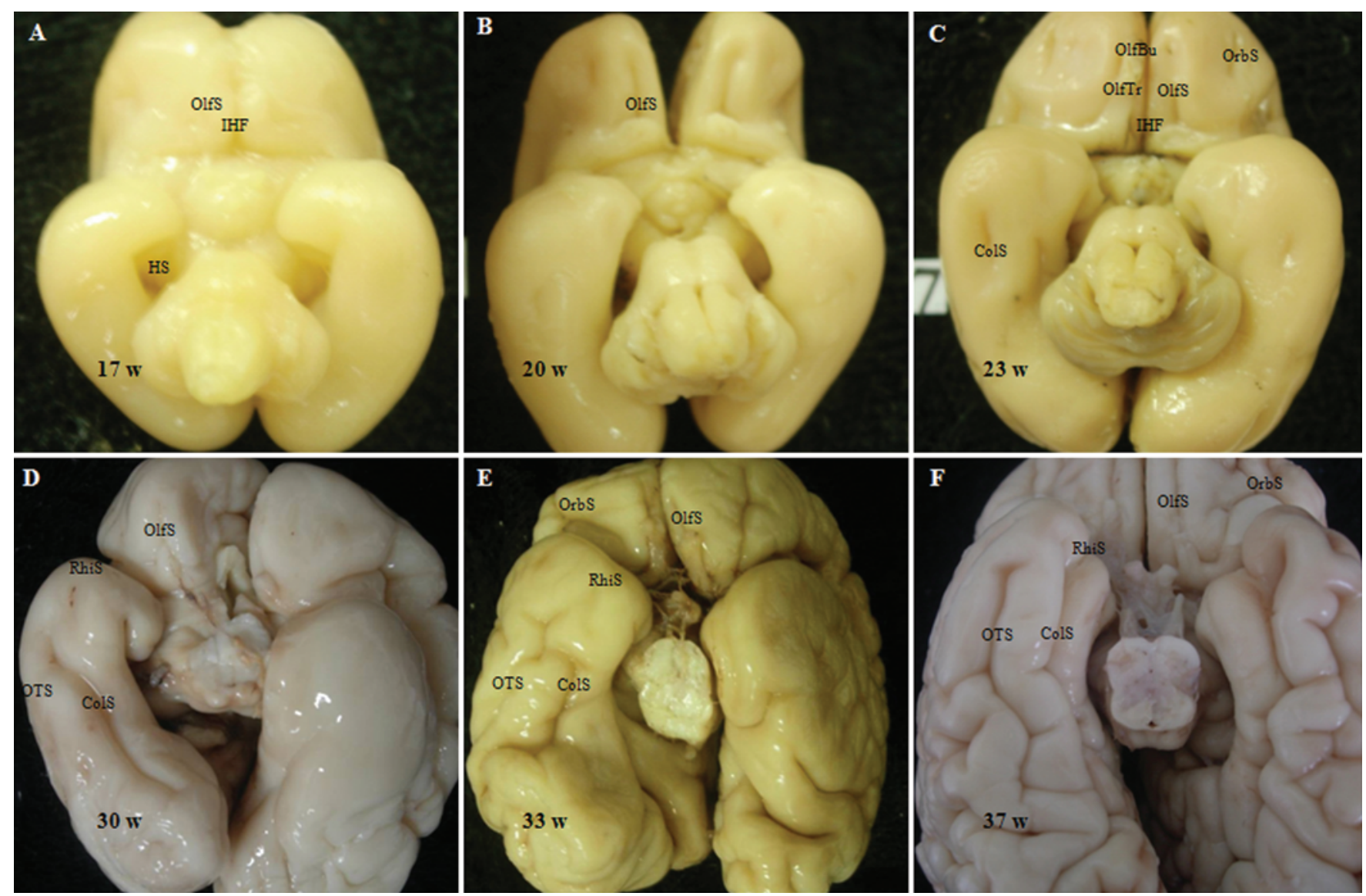

FIG. 3. Development of sulci of the inferior cerebral surface of the fetus at 17 (A), 20 (B), 23 (C), 30 (D), 33 (E), and 37 weeks (F). ColS = collateral sulcus; HS = hippocampal sulcus; OlfBu = olfactory bulb; OlfS = olfactory sulcus; OlfTr = olfactory tract; RhiS = rhinal sulcus.
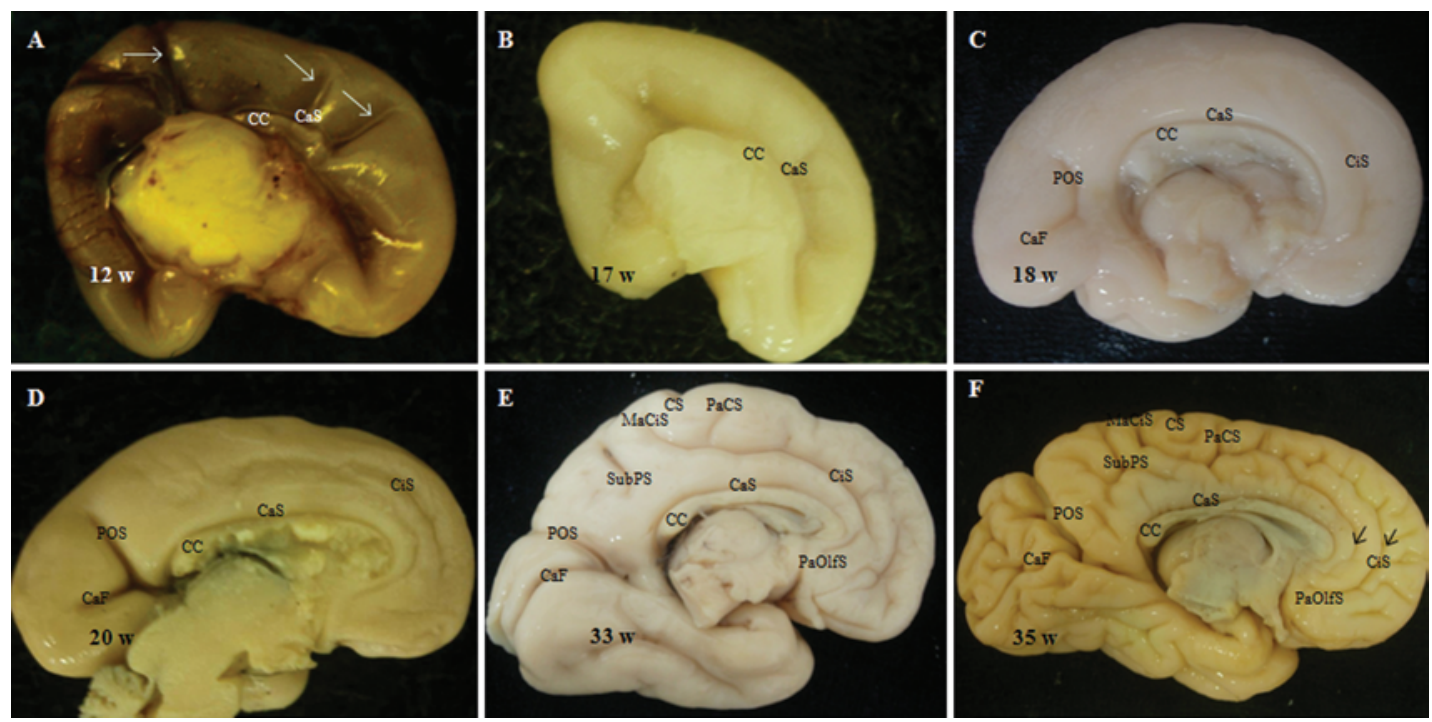

FIG. 4. Development of sulci of the medial cerebral surface of the fetus at 12 (A), 17 (B), 18 (C), 20 (D), 33 (E), and 35 weeks (F). The white arrows (A) indicate the appearance of transitory furrows during the initial phase of cerebral development. The black arrows $(\mathbf{F})$ indicate 2 parallel cingulate sulci. $\mathrm{CaF}=$ calcarine sulcus; $\mathrm{CaS}=$ callosal sulcus; $\mathrm{CC}=$ corpus callosum; $\mathrm{CiS}=$ cingulate sulcus; $\mathrm{MaCiS}=$ marginal branch of the cingulate sulcus; $\mathrm{PaCS}=$ paracentral sulcus; $\mathrm{PaOlfS}=$ paraolfactory sulcus; POS = parietooccipital sulcus (calcarine fissure); SubPS = subparietal sulcus. 
Study of the fetal morphological development of the brain sulci

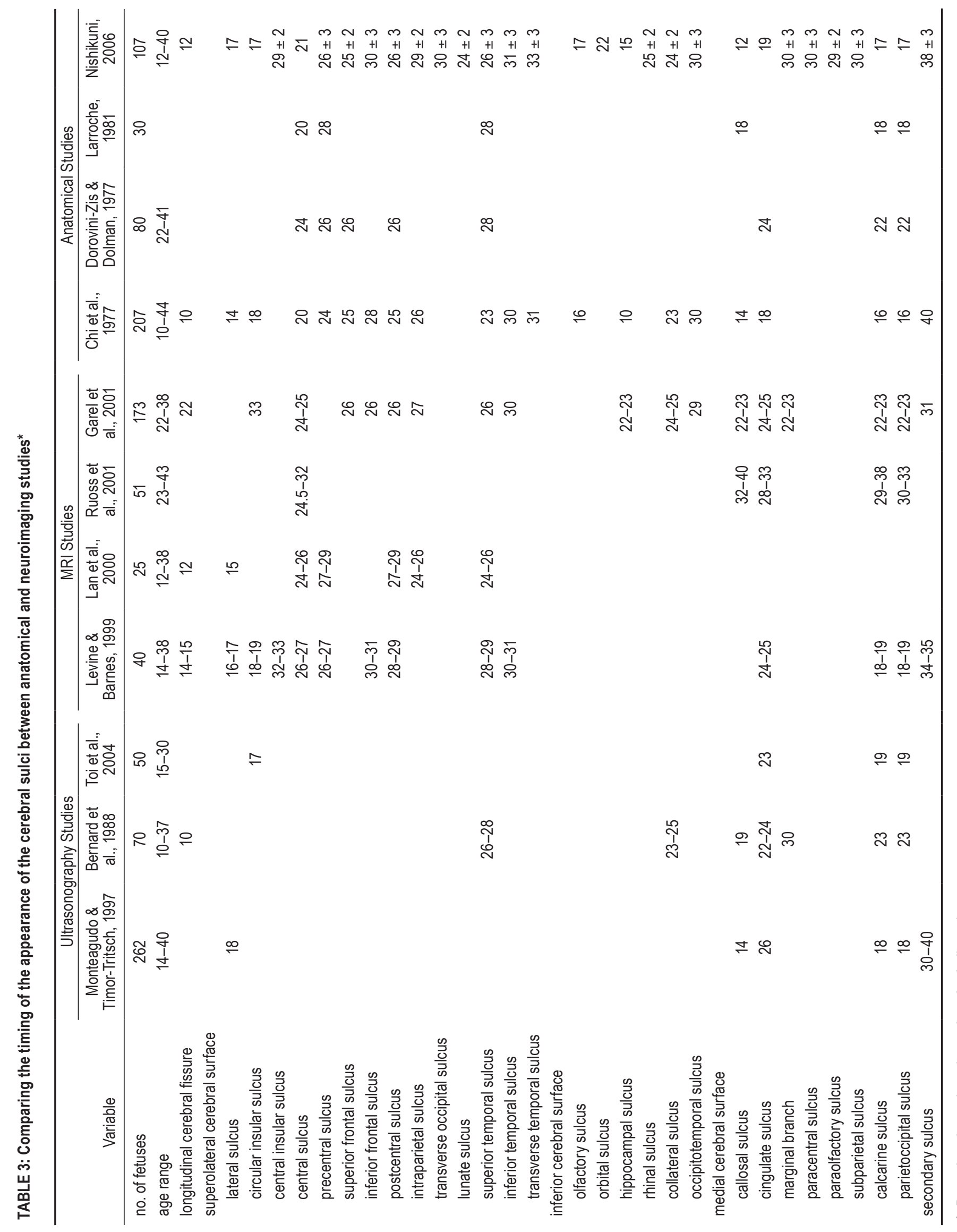


maturation, although there are discrepancies regarding the timing of the appearance of the cerebral sulci as observed by different authors. 5,7,14 These differences may be attributed to various factors: 1) gestational age, in which different criteria are used to calculate this factor (such as date of last menstruation, weight, height, cranial circumference, and occipital-sacral distance); 2) size of the examined sample; 3) technique, including gross visual inspection of the brain, photographs of fixed brains, and serial cuts of the brain; 4) side, including right and left hemispheres; and 5) inclusion of twin fetuses. Chi et al. ${ }^{5}$ observed that the superior frontal sulcus, the superior temporal sulcus, and the secondary sulci of the right cerebral hemisphere were visible 1-2 weeks before their appearance in the left hemisphere and that there was a delay of 2-3 weeks in the development of the sulci in the brains of twins.

Comparing our findings with the other equivalent anatomical studies available, it is apparent that we studied a larger number of sulci and that there are only small discrepancies between the common findings (Table 3). Comparing the timing of the appearance of the cerebral sulci between anatomical and neuroimaging studies, $1,5,7,11,12,14,17,18,28,34$ there is a 2- to 4-week delay between the appearance of the sulci in anatomical studies and visualization of the same sulci using ultrasonography; if MRI studies are used, the delay is 1 week.

\section{Conclusions}

The main aims of this anatomical study were to clarify the features of the development of the cerebral sulci and to find reliable morphological criteria for each gestational age. The cerebral surface is initially smooth, and after the development of transitory furrows, the permanent sulci appear initially as points or grooves, and then become depressions that gradually deepen. In the 33rd week of gestation, all of the primary sulci are already formed, and the secondary sulci then develop. The development of the cerebral sulci is gradual and proportional to the increasing body weight of the fetus.

\section{Disclosure}

The authors report no conflict of interest concerning the materials or methods used in this study or the findings specified in this paper.

Author contributions to the study and manuscript preparation include the following. Conception and design: both authors. Drafting the article: both authors. Critically revising the article: both authors. Reviewed submitted version of manuscript: both authors. Approved the final version of the manuscript on behalf of both authors: Nishikuni.

\section{References}

1. Bernard C, Droulle P, Didier F, Gérard H, Larroche JC, Plenat F, et al: [Echographic aspects of cerebral sulci in the ante- and perinatal period.] J Radiol 69:521-532, 1988 (Fr)

2. Berry M, Rogers AW: The migration of neuroblasts in the developing cerebral cortex. J Anat 99:691-709, 1965
3. Boling W, Olivier A, Bittar RG, Reutens D: Localization of hand motor activation in Broca's pli de passage moyen. J Neurosurg 91:903-910, 1999

4. Broca P: Sur la topographie crânie-cerébrale ou sur les rapports anatomiques du crâne et du cerveau. Rev d'Anthrop 5: $193-248,1876$

5. Chi JG, Dooling EC, Gilles FH: Gyral development of the human brain. Ann Neurol 1:86-93, 1977

6. Dooling EC, Chi JG, Gilles FH: Telencephalic development: changing gyral patterns, in Gilles FH, Leviton A, Dooling EC (eds): The Developing Human Brain: Growth and Epidemiologic Neuropathology. Boston: John Wright, 1983, pp 94-104

7. Dorovini-Zis K, Dolman CL: Gestational development of brain. Arch Pathol Lab Med 101:192-195, 1977

8. Duvernoy HM: The Human Brain. Wien: Springer, 1991

9. Encha-Razavi F, Sonigo P: Features of the developing brain. Childs Nerv Syst 19:426-428, 2003

10. Finger S: Origins of Neuroscience. New York: Oxford University, 1994

11. Garel C, Chantrel E, Brisse H, Elmaleh M, Luton D, Oury JF, et al: Fetal cerebral cortex: normal gestational landmarks identified using prenatal MR imaging. AJNR Am J Neuroradiol 22:184-189, 2001

12. Lan LM, Yamashita Y, Tang Y, Sugahara T, Takahashi M, Ohba T, et al: Normal fetal brain development: MR imaging with a half-Fourier rapid acquisition with relaxation enhancement sequence. Radiology 215:205-210, 2000

13. Larroche JC: The development of the central nervous system during intrauterine life, in Falkner F (ed): Human Development. Philadelphia: WB Saunders, 1966, pp 257-276

14. Larroche JC: Morphological criteria of central nervous system development in the human foetus. J Neuroradiol 8:93-108, 1981

15. Larroche JC: Quelques aspects anatomiques du développement cerebral. Biol Neonat 4:126-159, 1962

16. Levene MI, Lilford RJ: Fetal and Neonatal Neurology and Neurosurgery, ed 2. New York: Churchill Livingstone, 1995

17. Levine D, Barnes PD: Cortical maturation in normal and abnormal fetuses as assessed with prenatal MR imaging. Radiology 210:751-758, 1999

18. Monteagudo A, Timor-Tritsch IE: Development of fetal gyri, sulci and fissures: a transvaginal sonographic study. Ultrasound Obstet Gynecol 9:222-228, 1997

19. Naidich TP, Valavanis AG, Kubik S: Anatomic relationships along the low-middle convexity: Part I-Normal specimens and magnetic resonance imaging. Neurosurgery 36:517-532, 1995

20. Nishikuni K: [Study of the fetal and post-natal morphological development of the sulci of the brain. \{thesis\}] São Paulo: Faculdade de Medicina, Universidade de São Paulo, 2006 (Portuguese)

21. Ono M, Kubik S, Abernathey CD: Atlas of the Cerebral Sulci. New York: Thieme, 1990

22. Pearce JM: Louis Pierre Gratiolet (1815-1865): the cerebral lobes and fissures. Eur Neurol 56:262-264, 2006

23. Régis J, Mangin JF, Ochiai T, Frouin V, Riviére D, Cachia A, et al: "Sulcal root" generic model: a hypothesis to overcome the variability of the human cortex folding patterns. Neurol Med Chir (Tokyo) 45:1-17, 2005

24. Ribas GC: The cerebral sulci and gyri. Neurosurg Focus 28(2):E2, 2010

25. Ribas GC: [Considerations about the nervous system phylogenetic evolution, behavior, and the emergence of consciousness.] Rev Bras Psiquiatr 28:326-338, 2006 (Portuguese)

26. Ribas GC: [Surgical anatomy of microneurosurgical sulcal key-points. \{thesis\}] São Paulo: Faculdade de Medicina, Universidade de São Paulo, 2005 (Portuguese)

27. Ribas GC, Yasuda A, Ribas EC, Nishikuni K, Rodrigues AJ 


\section{Study of the fetal morphological development of the brain sulci}

Jr: Surgical anatomy of microneurosurgical sulcal key points. Neurosurgery 59 (4 Suppl 2):ONS177-ONS211, 2006

28. Ruoss K, Lövblad K, Schroth G, Moessinger AC, Fusch C: Brain development (sulci and gyri) as assessed by early postnatal MR imaging in preterm and term newborn infants. Neuropediatrics 32:69-74, 2001

29. Sarnat HB, Netsky MG: Evolution of the Nervous System, ed 2. New York: Oxford University Press, 1981

30. Squire LR (ed): Fundamental Neuroscience, ed 2. Amsterdam: Academic Press, 2003

31. Stocker JT, Dehner LP (eds): Pediatric Pathology, ed 2. Philadelphia: Lippincott Williams \& Wilkins, Vol 2, 2001

32. Tamraz JC, Comair YG: Atlas of Regional Anatomy of the Brain Using MRI. Berlin: Springer, 2000

33. Testut L: Tratado de anatomia humana, ed 8. Barcelona: Salvat, 1934

34. Toi A, Lister WS, Fong KW: How early are fetal cerebral sulci visible at prenatal ultrasound and what is the normal pattern of early fetal sulcal development? Ultrasound Obstet Gynecol 24:706-715, 2004

35. van der Knaap MS, van Wezel-Meijler G, Barth PG, Barkhof
F, Adèr HJ, Valk J: Normal gyration and sulcation in preterm and term neonates: appearance on MR images. Radiology 200:389-396, 1996

36. Williams PL, Warwick R (eds): Gray's Anatomy, ed 36. Philadelphia: Saunders, 1980

37. Yaşargil MG: Microneurosurgery, Vol 4. Stuttgart: Thieme, 1994

38. Yaşargil MG, Cravens GF, Roth P: Surgical approaches to "inaccessible" brain tumors. Clin Neurosurg 34:42-110, 1988

39. Yaşargil MG, Kasdaglis K, Jain KK, Weber HP: Anatomical observations of the subarachnoid cisterns of the brain during surgery. J Neurosurg 44:298-302, 1976

Manuscript submitted March 8, 2012.

Accepted September 25, 2012.

Please include this information when citing this paper: published online November 9, 2012; DOI: 10.3171/2012.9.PEDS12122.

Address correspondence to: Koshiro Nishikuni, M.D., Rua Castro Alves 654, Edifício Esmeralda 51, São Paulo, Brazil CEP $01532-$ 000. email: nishikuni@terra.com.br. 